\title{
Normalized Hilbert Huang Transform-NHHT Applied to Phase Extraction in Wavelet Domain
}

\author{
K. Assid, F. Alaoui, V. Dembele, S. Houmairi, M. Sidki and A. Nassim* \\ Laboratoire IMC- OTIS Optique \& Traitement Image et Signal; Université Chouaib Doukkali -FSJ; BP 20, El Jadida \\ 24000, Morocco
}

\begin{abstract}
The purpose of the present work is the use of the Normalized Hilbert-Huang Transform (NHHT) to generate an exact quadrature fringe pattern. The NHHT has helped to overcome many of the difficulties of the regular Hilbert transform. In the wavelet domain, the phase evaluation method needs a fringe pattern with a spatial carrier. It is possible to provide synthetically a modulated fringe pattern by combining a computer generated grating with the pattern and its quadrature. Then, using continuous wavelet transform decomposition, it is easy to calculate the phase gradient computed from maximums scales and provide by simple integration the phase map coded in the fringe pattern. This method leads directly to phase distribution from a single pattern avoiding the complex step of phase unwrapping.
\end{abstract}

Keywords: Normalized Hilbert Huang Transform (NHHT), Empirical Mode Decomposition (EMD), Phase Evaluation Methods, Continuous Wavelet Transform (CWT).

\section{INTRODUCTION}

Many whole field optical techniques provide the measurement data codified as the phase of a periodic intensity profile. The obtained image is called a fringe pattern. The fringe pattern analysis generally consists of the phase evaluation methods [1,2] employed to measure various physical magnitudes in variety of scientific and engineering fields. The phase evaluation methods can be classified as methods without a spatial carrier such as the phase shifting method [3] and methods with a spatial carrier such as the Fourier transform method [4] and the wavelet method [5,6]. Using just a single fringe pattern, the phase evaluation methods with a spatial carrier are adequate for real time acquisition and dynamic process.

It is possible to provide a modulated fringe pattern by the superposition of a computer-generated grating with two $\pi / 2$ shifted patterns [7]. The spatial carrier is then, introduced digitally by combining the pattern and its quadrature; the entire process is carried out directly in the computer. Wavelet transform decomposition [8] leads directly to phase distribution avoiding the complex step of phase unwrapping.

Considering that significant improvement has been made since 1998 [9-11], in this study we applied the Normalized Hilbert-Huang Transformation (NHHT) to generate an exact quadrature fringe pattern. The NHHT is a novel digital signal processing technology based on the combination of the empirical mode decomposition (EMD), normalization and Hilbert transform application. The NHHT has helped to overcome many of the difficulties of the regular Hilbert transform. It was developed to satisfy the specific limitations

*Address correspondence to this author at the Laboratoire IMCInstrumentation de Mesure \& Contrôle. Equipe OTIS: Optique \& Traitement Image et Signal.Université Chouaib Doukkali -FSJ : BP 20, El Jadida 24000, Morocco; Tel: (212) 523342325; Fax: (212) 523342187;

E-mail: knassim58@gmail.com set by the Bedrosian and Nuttall theorems [12]. The comprehensive review can be found in [13].

The paper is organized as follows. Section 2 contains a short presentation of the Normalized Hilbert-Huang Transform applied to optical fringe pattern. We then expose in section 3 the synthetic modulated fringe pattern. Section 4 is devoted to phase evaluation by wavelet transform analysis. Finally, results and conclusion are presented in section 5 .

\section{QUADRATURE FRINGE PATTERN GENERATION BY NHHT}

The fringe patterns, derived from two-beam interferometers, is formulated by the sinusoidal dependence of the intensity on the spatial coordinates $(x, y)$ of the image plane

$I(x, y)=a(x, y)+b(x, y) \cos \varphi(x, y)$

Where $\mathrm{a}(\mathrm{x}, \mathrm{y})$ is the background. The terms $\mathrm{b}(\mathrm{x}, \mathrm{y})$ and $\varphi(\mathrm{x}, \mathrm{y})$ are the visibility function and the optical phase.

The empirical mode decomposition (EMD) analysis is an adaptive and fully-driven data method to decompose any signal into a set of components called intrinsic mode functions (IMF) and a residue. Adding all the IMFs together with the residue reconstructs the original signal without information loss or distortion.

When EMD is applied to a fringe pattern and adding all the IMFs by just skipping the DC component we remove the background. The filtered fringe pattern is

$$
\begin{aligned}
& J(x, y)=\sum_{i=1}^{n-1} I M F_{i}(x, y) \\
& J(x, y)=b(x, y) \cos \varphi(x, y)
\end{aligned}
$$

An IMF is not restricted to a narrow band signal, and it can be both amplitude and frequency modulated, otherwise the AM variations will contaminate the FM part. The 
normalization step is designed to render IMFs into the AMFM model and separate the AM and FM parts by taken the envelope as the modulus of the analytic signal of IMF. Huang and Long have proposed the normalization of the IMFs in the following steps: Starting from an IMF, they first find all the maxima of the IMFs, defining the envelope by spline through all the maxima. Now, normalize the IMF by dividing the IMF by the envelope. Thus, they have the normalized function having amplitude always equal to unity, and have circumvented the limitation of Bedrosian theorem.

$$
J_{n}(x, y)=\sum_{i=1}^{n-1} N I M F_{i}(x, y)
$$

The quadrature signal is obtained by the combination of the Hilbert spectrum of each normalized IMF instead of a direct Hilbert spectrum on IMF due to the Bedrosian theorem. The Hilbert Transform (HT) is the convolution product of the signal and $1 / \pi \mathrm{x}$. The quadrature fringe patterns is

$$
\begin{aligned}
& J_{q}(x, y)=\sum_{i=1}^{n-1} H T\left[\operatorname{NIMF}_{i}(x, y)\right] \\
& J_{q}(x, y)=b(x, y) \sin \varphi(x, y)
\end{aligned}
$$

\section{SYNTHETIC MODULATED FRINGE PATTERN}

The requirement for appropriately introducing a spatial carrier into a fringe pattern is a difficult process. In order to permit measurement with a greater phase variation, the spatial carrier is introduced digitally by combining the pattern and its quadrature. The entire process is carried out directly in the computer. The fringe pattern and its Quadrature are defined by

$$
\begin{aligned}
& J(x, y)=b(x, y) \cos \varphi(x, y) \\
& J_{q}(x, y)=\sum_{i=1}^{n-1} H T\left[N \operatorname{NMF}_{i}(x, y)\right]
\end{aligned}
$$

The modulated fringe pattern is obtained by combining a computer-generated grating to the two shifted patterns

$$
\begin{aligned}
& J_{c}(x, y)=J(x, y) \cdot \cos (c x)-J_{q}(x, y) \cdot \sin (c x) \\
& J_{c}(x, y)=b(x, y) \cos (c x+\varphi(x, y))
\end{aligned}
$$

The frequency carrier $\mathrm{c}$ is selected such that satisfying the condition

$$
c>|\partial \varphi / \partial x|_{\max }
$$

The carrier period c can be easily varied. The carrier frequency is not critical factor because the grating period is generated after the pattern has been acquired and can be modified in a simple manner.

\section{WAVELET OPTICAL PHASE EXTRACTION}

Wavelets offer a powerful method to quantify how energy is spatially distributed at multiple scales [14]. A good review of wavelet theory has been published by Daubechies [15]. The one-dimensional wavelet transform of the line $y$ in the modulated fringe pattern, in the $\mathrm{x}$ direction, is given by $w(s, u)=s^{-1 / 2} \int_{-\infty}^{+\infty} J_{c}(x) \cdot \psi^{*}((x-u) / s) \cdot d x$

And the Parseval identity leads to

$$
w(s, u)=(2 \pi)^{-1} \cdot s^{1 / 2} \cdot \int_{-\infty}^{+\infty} \hat{J}_{c}(k) \cdot \hat{\psi}^{*}(s k) \cdot \exp (i u k) \cdot d k
$$

where * denotes complex conjugation, $\psi((\mathrm{x}-\mathrm{u}) / \mathrm{s})$ is the analyzing wavelet obtained by shifting and scaling the admissible and localized mother wavelet $\psi(x) ; s>0$ is the scale parameter related to the frequency concept, and $u$ is the shift parameter related to position. Jc is the modulated fringe pattern.

The $w(s, u)$ coefficients quantify the similitude between the modulated fringe pattern $\mathrm{Jc}(\mathrm{x})$ and the wavelet $\psi((\mathrm{x}-\mathrm{u}) / \mathrm{s})$. For a given scale $\mathrm{s}$ at a position $\mathrm{u}$, the magnitude of this resemblance is given by the modulus of w(s, $u)$. The $\mathrm{w}(\mathrm{s}, \mathrm{u})$ is also regarded as coordinates of the signal projected in the wavelet basis formed by $\psi((\mathrm{x}-\mathrm{u}) / \mathrm{s})$ set.

Exploiting the localization property of the wavelet, and the development of the phase of interest $\varphi$ on Taylor series close to the central value, we assume a slow variation of $\mathrm{b}(\mathrm{x}, \mathrm{y})$ over the support of the wavelet, which is convenient in usual cases. Owing to the localization of the wavelet, we can neglect the higher order of $(x-u)$. With these considerations, the wavelet transform becomes

$$
w(s, u)=s^{-1 / 2} \int_{-\infty}^{+\infty} \cos (\phi(u)+(x-u) \cdot p(x)) \cdot \psi^{*}((x-u) / s) \cdot d x
$$

Where

$$
p=(c+\partial \varphi(x) / \partial x)
$$

And the Parseval identity leads to

$$
w(s, u)=0.5 s^{1 / 2} \cdot \int_{-\infty}^{+\infty} h(k) \cdot \hat{\psi}^{*}(s k) \cdot \exp (i u k) \cdot d k
$$

Where

$$
\begin{aligned}
& h(k)=h_{1}(k)+h_{2}(k) \\
& h_{1}(k)=\delta(k-p(u)) \exp (i(\phi(u)-u \cdot p(u)) \\
& h_{2}(k)=\delta(k+p(u)) \exp (-i(\phi(u)-u \cdot p(u)))
\end{aligned}
$$

The wavelet transform becomes

$$
w(s, u)=0.5 s^{1 / 2} \cdot\left[\hat{\psi}^{*}(s \cdot p) e^{i \phi(u)}+\hat{\psi}^{*}(-s \cdot p) e^{-i \phi(u)}\right]
$$

The mother wavelet $\psi$ is the second order of Paul wavelet [16] which is formulated by

$\psi(x)=\frac{2^{n} n !(1-i x)^{-(n+1)}}{2 \pi \sqrt{(2 n) ! / 2}}$

Where $\mathrm{n}$ is the order of Paul wavelet.

Its Fourier transform FT is

$\hat{\psi}(k)=\frac{2^{n}}{\sqrt{n(2 n-1) !}} k^{n} \exp (-k) H(k)$ 
where $\mathrm{H}$ is the Heaviside distribution

In the case of Paul mother wavelet, its Fourier transform is null in the negative frequencies, so the second term can be neglected and the modulus can expressed as

$w m(s, u)=\frac{p^{n}}{(2 n) !} \cdot s^{n+1 / 2} \cdot \exp (-s \cdot p)$

The phase gradient is computed from extremums scales $\mathrm{q}$ (u, y) by

$\partial \varphi(u, y) / \partial x=-c+(2 n+1) / 2 q(u, y)$

This leads to the phase by integration of the gradient.

\section{RESULTS AND CONCLUSION}

The numerical simulation consists in generating digitally a fringe pattern to verify the ability of the method to determine the phase distribution. The test phase function we used is

$\varphi(x, y)=0.0005 \cdot\left[(x-256)^{2}+(y-256)^{2}\right]$

The intensity distribution of fringe pattern shown in Fig. (1) is

$I(x, y)=1+0.5 \cos \varphi(x, y)$

In Fig. (2) we illustrated the IMF's of the fringe pattern from EMD decomposition. The quadrature fringe pattern

Fig. (1). The fringe pattern obtained by numerical simulation.
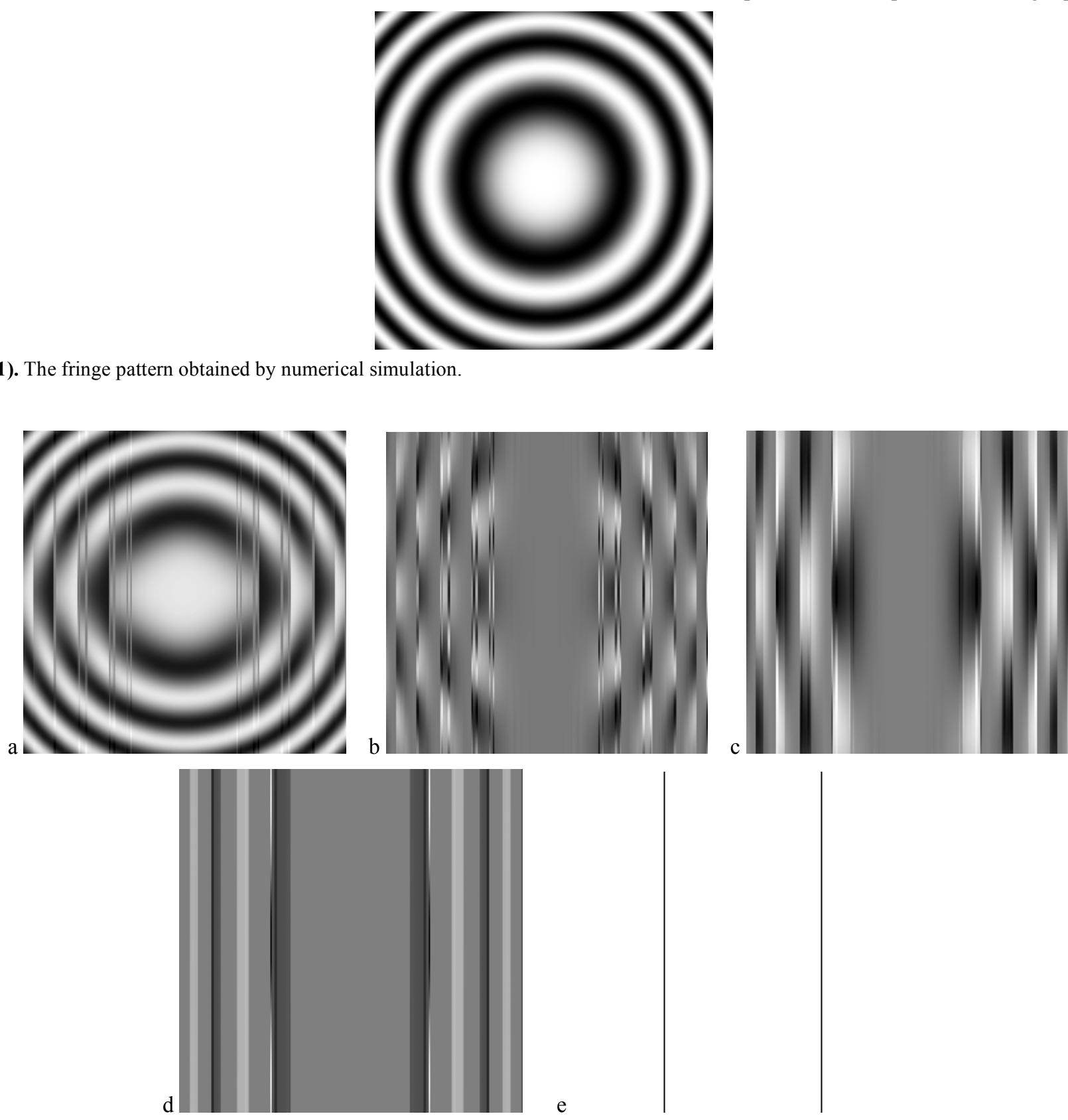

e

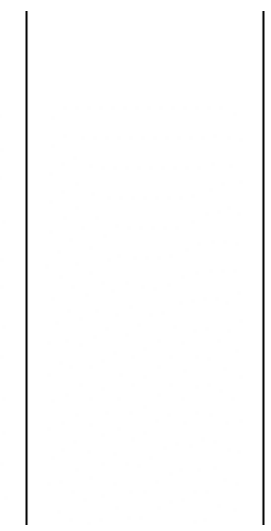

Fig. (2). The IMF's of the fringe pattern obtained by EMD decomposition. 
obtained by normalized Hilbert Huang transforms NHHT is depicted in Fig. (3). In Fig. (4) we illustrated the quadrature fringe pattern obtained by normalized Hilbert Huang transforms. In Fig. (5) we present the modulated fringe pattern obtained by combining numerically the fringe pattern and its quadrature. The carrier frequency of the computer-generated grating is $\mathrm{c}=1.08 \mathrm{rd} / \mathrm{pixel}$ (the maximum gradient of the phase $\varphi$ is $0.74 \mathrm{rad} / \mathrm{pixel}$ ). The carrier period can be easily varied. The difference between the test phase and the retrieved phase from wavelet method is presented Fig. (6). In conclusion, our method provides the optical phase distribution from a single fringe pattern with a high accuracy. This method is adequate for real time acquisition and easy to implement and the high potential accuracy is attained with a conveniently frequency carrier selection.

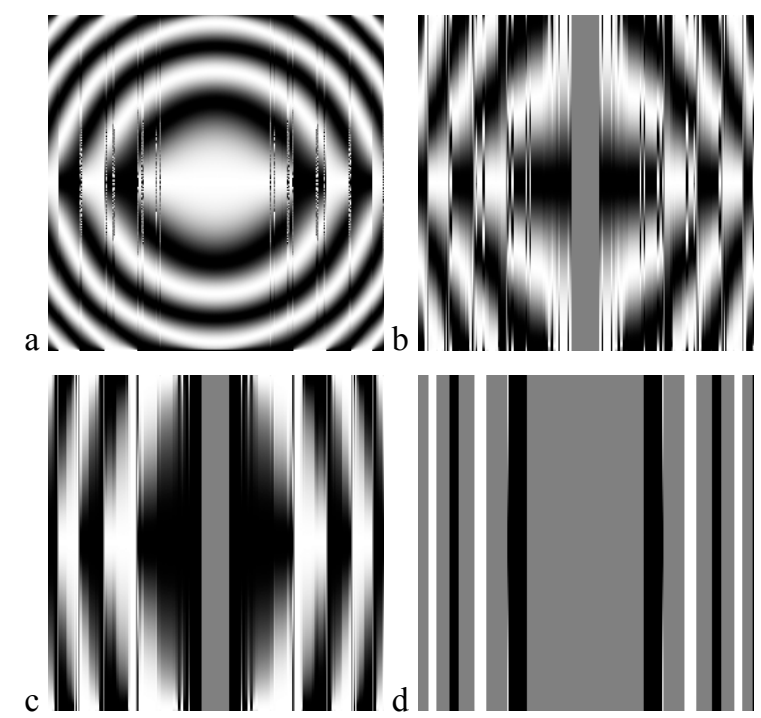

Fig. (3). The normalized IMF's of the fringe pattern.

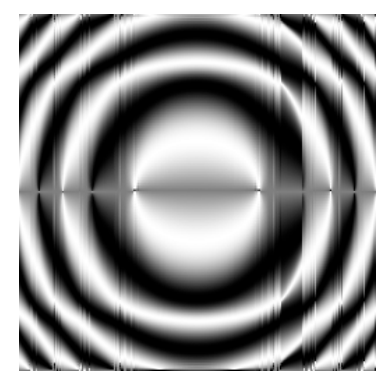

Fig. (4). The quadrature fringe pattern obtained by normalized Hilbert Huang transforms.

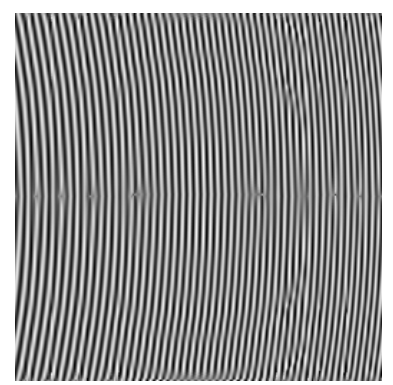

Fig. (5). The modulated fringe pattern obtained digitally from the fringe pattern and its quadrature.

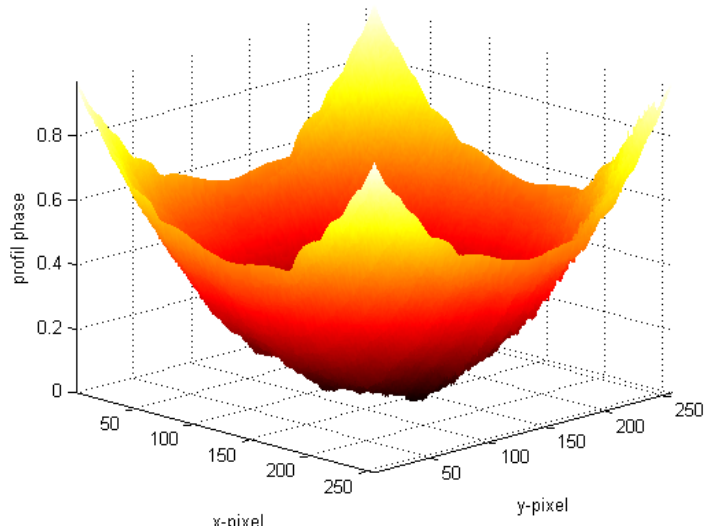

$\mathrm{a}$

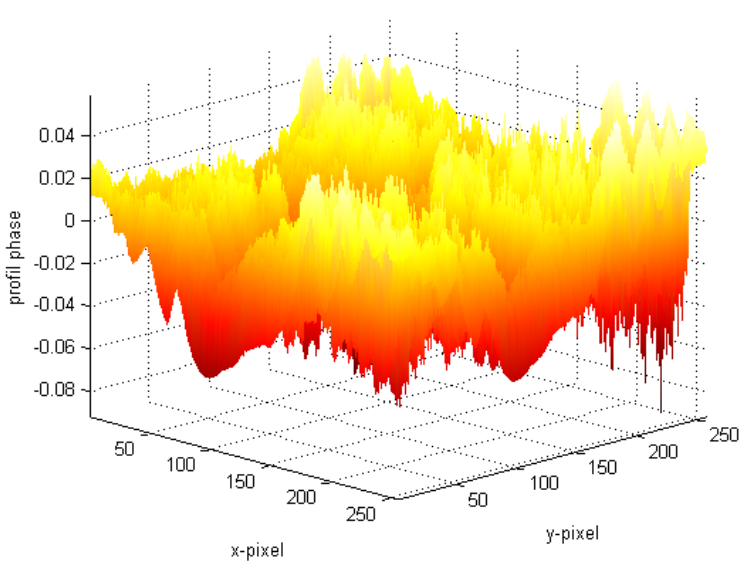

b

Fig. (6). The difference between the test phase and the phase from wavelet method.

\section{REFERENCES}

[1] Sirohi SR, Chau SF. Optical methods of measurement. Marcel Dekker Inc 1999.

[2] Dorrio VB, Fernandez LL. Phase- evaluation methods in wholefields optical measurement techniques. Meas Sci Technol 1999; 10: 33-55.

[3] Creath K. Phase measurement Interferometry techniques. Prog Opt 1988; 26: 349-93.

[4] Tageda MS, Kobayashi S. Fourier transform methods of fringe pattern analysis for computer based topography and Interferometry. Opt Soc Am 1982; 72: 1156-60.

[5] Afifi M, Fassi FA, Marjane M, Nassim K, Sidki M, Rachafi S. Paul wavelet based algorithm for optical phase distribution evaluation. Opt Commun 2002; 211: 47-51.

[6] Bahich M, Afifi M, Barj EM. Optical phase extraction algorithm based on the continuous wavelet and the Hilbert transform. J Comput 2010; 2: 5 .

[7] Barj EM, Afifi M, Idrissi AA, Rachafi S, Nassim A. A digital spatial carrier for wavelet phase extraction. Opt Int J Light Electron Opt 2005; 116: 507-10.

[8] Daubechies I. The wavelet transform time-frequency localization and signal analysis. IEEE Trans Inf Theory 1990; 36: 961-1005.

[9] Huang NE, Long SR. A generalized zero crossing for local frequency determination. U.S. Patent pending 2003. 
[10] Huang NE, Shen Z, Long SR, et al. Mathematical physical and engineering sciences (A454): Proc R Soc, Lond 1998; 454(1971): 903-95.

[11] Huang NE, Shen Z, Long SR. A new view of nonlinear water waves: the Hilbert spectrum. Fluid Mech 1999; 31: 417-57.

[12] Nuttall AH, Bedrosian E. On the quadrature approximation to the Hilbert transform of modulated signals (51): Proceeding of the IEEE 1963.
[13] Huang NE, Wu Z. A review on Hilbert-Huang transform: Method and its applications to geophysical studies. Geophys 2008; RG2006 23PP.

[14] Kaiser G. A Friendly Guide to Wavelets. Birkhauser: Boston; 1994

[15] Daubechies I. Ten Lectures on Wavelets. Society for Industrial and Applied Mathematics: Philadelphia 1992.

[16] Torrence C, Compo GP. A practical guide to wavelet analysis. Bull Am Meteorol Soc 1998; 79: 61-78.

(C) Assid et al.; Licensee Bentham Open.

This is an open access article licensed under the terms of the Creative Commons Attribution Non-Commercial License (http://creativecommons.org/ licenses/by-nc/3.0/), which permits unrestricted, non-commercial use, distribution and reproduction in any medium, provided the work is properly cited. 